\title{
Direct Laser Writing: Versatile Tool for Microfabrication of Lithium Niobate
}

\author{
Vygantas MIZEIKIS $^{* 1}$, Vytautas PURLYS ${ }^{* 2}$, Domas PAIPULAS ${ }^{* 2}$, Ričardas BUIVIDAS ${ }^{* 3}$ and Saulius JUODKAZIS ${ }^{* 3}$ \\ ${ }^{* 1}$ Division of Global Research Leaders (Research Institute of Electronics), Shizuoka University, \\ 3-5-3-1 Johoku, Naka-ku, Hamamatsu 432-8561, Japan \\ E-mail:dvmzks@ipc.shizuoka.ac.jp \\ *2 Laser Research Center, Department of Quantum Electronics, \\ Vilnius University, Vilnius, Lithuania \\ ${ }^{* 3}$ Centre for Micro-Photonics, Swinburne University of Technology, Melbourne, Australia
}

\begin{abstract}
We demonstrate versatility of femtosecond direct laser writing (DLW) technique for microstructuring of iron-doped and as-grown lithium niobate crystals. Formation of extended, metastable, and re-writable refractive index modulation structures is achievable via fast local photorefractive modification of refractive index at the focus of the writing laser beam, with a modulation of up to $\Delta \mathrm{n} \sim 10^{-3}$, without permanent structural damage to the host crystal. Non-destructive photomodification allows total or partial erasure or modification of the previously recorded structures. This capability may enable realization of optically reconfigurable diffractive optical elements in the same volume of lithium niobate crystal. Flexibility of the DLW technique can be exploited for threedimensional microstructuring of periodic and aperiodic patterns with controllable index modulation profiles.
\end{abstract}

DOI:10.2961/jlmn.2012.03.0020

Keywords: Lithium niobate, direct laser writing technique, diffractive optical elements, photorefractive effect

\section{Introduction}

Lithium niobate $\left(\mathrm{LiNbO}_{3}\right)$ is widely used in modern photonics due to its attractive physical properties, such as ferroelectricity, piezoelectricity, photoelesticity, uniaxial birefringency, optical nonlinearities [1], and a wide spectral window of optical transparency within the $0.3-5 \mu \mathrm{m}$ wavelength range. Bulk lithium niobate is used in optical modulators, Q-switches, and optical harmonics generators. Microstructuring of lithium niobate has enabled creation of optical waveguides, optical memories, narrow-band spectral filters, and diffractive optical elements (DOE) [13]. Microstructuring techniques aimed at creation of localized regions with modified refractive index, traditionally use planar masks on the surface of crystal for creation of modified regions via proton exchange or titanium in-diffusion[1]. Optical waveguides, interconnects, interferometers, and optical networks can be realized using this approach. The microstructuring is permanent and can not be modified subsequently. Holographic recording by exposure of doped $\mathrm{LiNbO}_{3}:(\mathrm{Fe}, \mathrm{Mn}, \mathrm{Zn})$ crystals to periodic interference patterns of two or more coherent laser beams offers the possibility of all-optical recording of refractive index modulation patterns in the bulk of the crystal, and is widely used for formation of narrowband spectral filters and holographic memories[4]. This non-contact optical microstructuring technique exploits index modulation induced using bulk photovoltaic effect. The photomodification is reversible, and can be erased optically. However, holographic recording allows recording of periodic patterns only, and requires lengthy exposures. Here we demonstrate application of femtosecond Direct Laser Writing (DLW) technique for microstructuring of $\mathrm{LiNbO}_{3}$. Using this approach, the advantage of noncontact, non-destructive photomodification mechanism can be combined with the capability of the DLW technique to write almost arbitrary structures in 1D, 2D, and 3D. Photorefractive modification of iron-doped lithium niobate by tightly focused femtosecond pulses is illustrated schematically in Fig. 1. Photoexcitation of free mobile carriers from stationary dopant iron centers occurs in the focal region via non-linear absorption. Photoexcited carriers diffuse away from excitation sites and become trapped at other dopants/defects outside of the photoexcited region. This results in formation of space charge field, and local modulation of the refractive index via electro-optic effect, which is most pronounced along the crystallographic $c$-axis direction due to dominant value of the electro-optic coefficient along this direction. This mechanism is essentially identical to that exploited in holographic recording [4]. Qualitative spatial index modulation profile induced by focused laser beam is shown schematically in Fig. 3(a)[5,6]. Translation of the focal spot allows formation of extended refractive index modulation structures in the bulk of the sample. Previous applications of DLW technique for microstructuring of lithium niobate mostly exploited permanent destructive photomodification by high power laser pulses[7,8]. Use of non-destructive, reversible photorefractive photomodification for DLW in $\mathrm{LiNbO}_{3}$ has attracted less attention so far[9-11], and was 


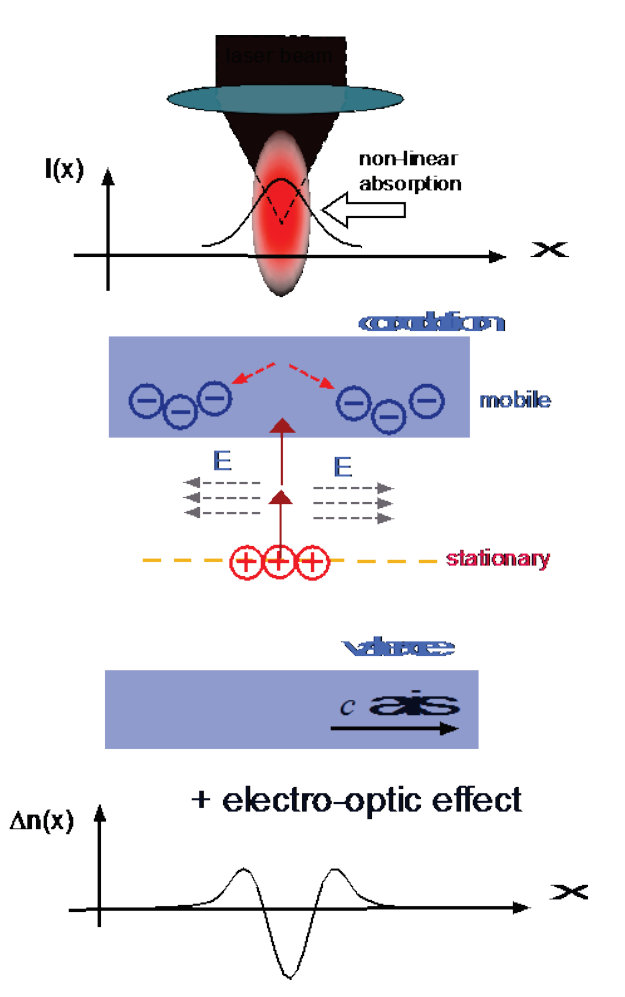

Fig. 1 Schematic explanation of photoexcitation of irondoped lithium niobate by infrared, tightly focused femtosecond laser pulses, and formation of local refractive index modulation via photorefractive effect.

mostly used for formation of optical memory structures. Here, we use DLW to create extended diffractive optical elements (DOE). The fabricated structures exhibit strong modulation of the refractive index approaching $\Delta \mathrm{n} \sim 10^{-4}$.. $10^{-3}$, and can be completely or partially erased and rewritten using focused laser beam. Such flexibility may prove useful for all-optical realization of adaptive diffractive elements in bulk of lithium niobate crystals.

\section{Experimental setup and samples}

Optical setup used for the DLW experiments is schematically depicted in Fig. 2. The laser source was derived from a Tsunami (Spectra Physics) femtosecond laser with a temporal pulse length of $130 \mathrm{fs}$, a central wavelength of $\lambda=800 \mathrm{~nm}$, and a repetition rate of $80 \mathrm{MHz}$. At this wavelength, lithium niobate crystals, although photosensitive near the $600-650 \mathrm{~nm}$ wavelength due to iron dopants, are completely transparent, and photoexcitation requires non-linear absorption. The laser beam was attenuated by a variable attenuator (not shown) and focused into the bulk of sample by a microscope objective lens. Lenses having various numerical apertures were used (NA=0.35, 0.4). DLW was facilitated by moving the sample using computer-controlled three-dimensional translation stage at a constant linear translation speed (typically in the range of 20-200 $\mu \mathrm{m} / \mathrm{s}$ ). The samples were slabs of $\mathrm{LiNbO}_{3}$ doped by $\mathrm{Fe}(400 \mathrm{ppm})$ and $y$-cut, i.e., with crystalline $c$-axis (along which photorefractive effect is most pronounced) was parallel to the polished top and bottom facets of the slabs. The DLW setup was assembled on the basis of Olympus IX71 inverted microscope, which allowed easy in situ observation of the fabrication process using condensor illumination and a video camera attached to

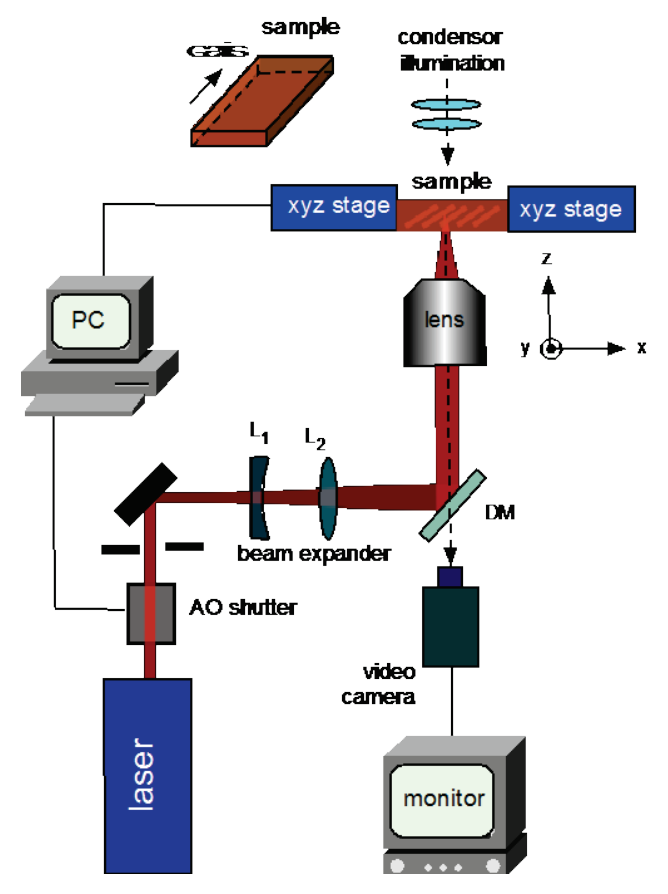

Fig. 2 Experimental setup for DLW fabrication

output port of the microscope. The microscope also provided basic post-fabrication readout of the recorded structures. The illuminating light was polarized along the $c$ axis in order to maximize the image contrast.

Previously DLW fabrication of lithium niobate used amplified laser source with low repetition rate of $(\sim 1000$ $\mathrm{Hz}$ ) and high pulse energy (up to microjoules)[9-11]. Such source was capable of inducing strong local photorefractive effect at the focus even by a single laser pulse. However, only a fraction (several nanojoules) of the available pulse energy (nearly one milijoule) could be exploited without inducing permanent damage to he crystal. In this respect, high repetition rate oscillator used in this work is advantageous, since its low pulse energy prevents permanent damage, and high repetition rate ensures smooth modification along the recorded linear features. While each laser pulse induces only minute refractive index changes in accordance to Fig. 1, large number of weak pulses can quickly augment index modulation comparable to that induced by a single powerful pulse.

\section{Results and discussion}

\subsection{Magnitude of refractive index modulation}

One-dimensional diffraction grating consisting of parallel straight lines is one of the simplest DOE easy to record and investigate. It also allows one to determine the magnitude of refractive index modulation from grating diffraction efficiency.

Figure 3(a) shows optical image of a diffraction grating with a lattice period of $\Lambda=13 \mu \mathrm{m}$, recorded using $\mathrm{NA}=0.35$ lens at an average laser power (measured before the lens) of $35 \mathrm{~mW}$, and sample translation speed of $100 \mu \mathrm{m} / \mathrm{s}$. Quality of the grating can be evidenced by its diffraction pattern 
of a
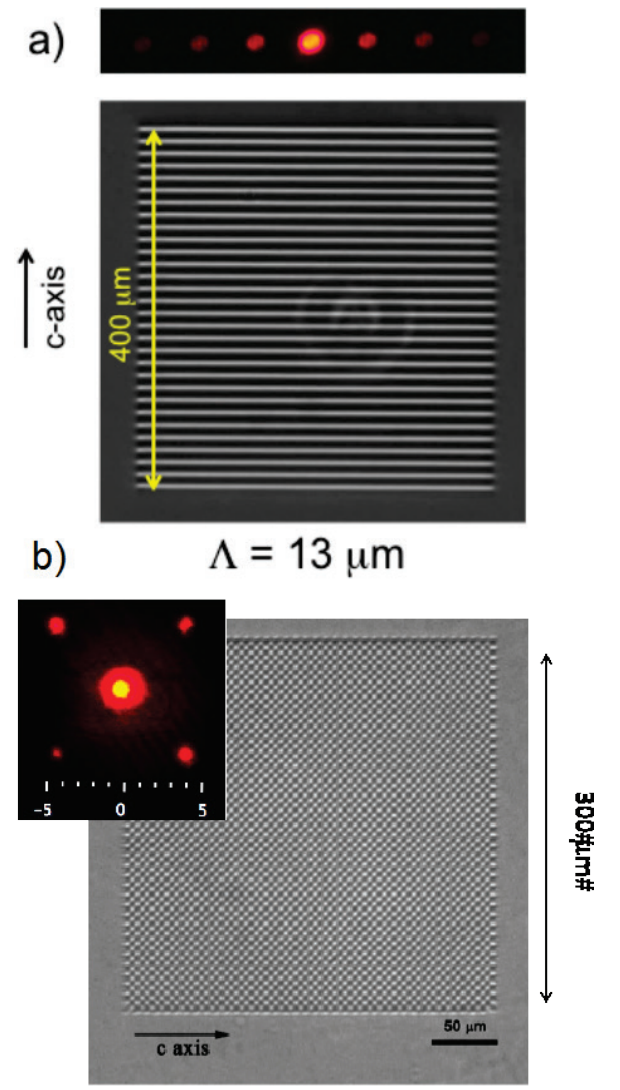

Fig. 3 Optical microscopy images of (a) one- and (b) twodimensional diffraction gratings. The insets show the corresponding diffraction patterns.

$\lambda=650 \mathrm{~nm} \quad \mathrm{cw}$ semiconductor laser beam shown in Fig. 3(a). The laser beam was focused about $50 \mu \mathrm{m}$ below the sample surface during recording, and was polarized linearly along the $c$-axis. According to qualitative in situ observations of the writing process, optical contrast of the written lines did not change when the angle between the linearly polarized writing beam and the $c$-axis was varied. This observation reflects the fact that refractive index modification arises due to the density gradient of photoexcited free carriers in the focal region. In the transverse plane (plane normal to the beam propagation) this gradient has nearly perfect radial symmetry regardless of the polarization of the writing beam, and thus laser writing is nearly independent of the beam polarization. On the other hand, optical contrast of recorded lines is strongly anisotropic, and is strongest when the lines are perpendicular to the crystalline $c$-axis, because photorefractive effect is strongest along the $c$-axis direction. Translation of the focal spot along the $c$-axis does not induce observable refractive index modulation. Nevertheless, it is possible to record various linear and curved features, as long as they have segments non-parallel to the $c$-axis direction. For-example, two-dimensional grating with period $\Lambda=13 \mu \mathrm{m}$ (recording conditions are the same as before) shown in Fig. 3(b) was recorded by drawing two perpendicular sets of parallel lines oriented at an angle of $45^{\circ}$ with respect to the $c$-axis.

We have estimated the magnitude of refractive index modulation from measured diffraction efficiency, assuming optically thin phase gratings with sinusoidal profile. Although geometrical thickness of the grating is considerable a)

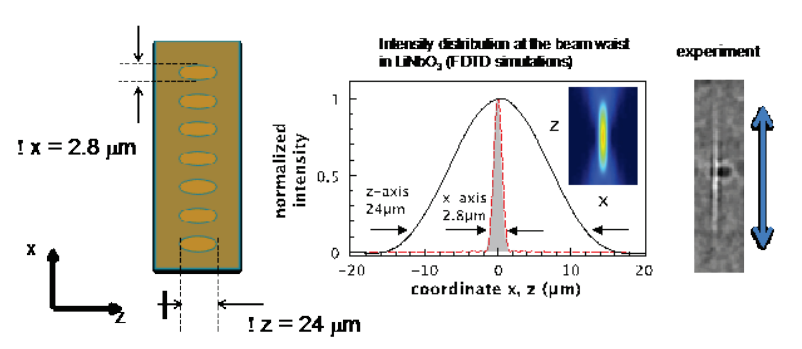

b)

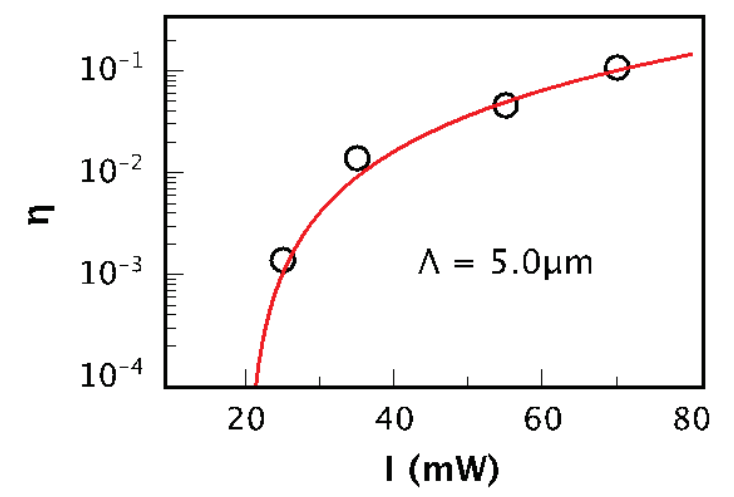

Fig. 4 Determination of refractive index modulation from the grating diffraction efficiency. (a) Structural parameters of the grating and comparison between calculated and measured geometrical thickness, (b) dependence of the diffraction efficiency on the laser power.

(see below), multiple positive and negative diffraction orders indicate that its optical thickness is too low for Bragg diffraction. In order to obtain harmonic index modulation profiles, a slightly shorter lattice period of $\Lambda=5 \mu \mathrm{m}$ was used. Various parameters of the gratings used for the estimates are explained schematically in Fig. 4(a).

First-order diffraction efficiency of a thin sinusoidal phase grating [12] can be expressed as

$$
\eta=\mathrm{J}_{1}^{2}(\Delta \phi)
$$

where $\mathrm{J}_{1}$ is Bessel function of the first kind, and $\Delta \phi$ is the phase difference due to the refractive index modulation. The phase difference can be approximated as

$$
\Delta \phi=\frac{2 \pi \Delta n \Delta z}{\lambda}
$$

where $\Delta \mathrm{n}$ is the refractive index modulation, $\Delta \mathrm{z}$ is the geometrical thickness of grating, and $\lambda$ is the probing wavelength. To understand idealized parameters of gratings drawn by a focused laser beam, theoretical modeling of the beam intensity distribution at the focus was done using Finite-Difference Time-Domain (FDTD) simulations. The parameters used in the simulations (laser wavelength, NA of the focusing lens, refractive index of the crystal) were the same as the experimental values. Consequently, the focal spot size is estimated to be approximately $2.8 \mu \mathrm{m}$ and $24 \mu \mathrm{m}$ in the lateral and axial directions, respectively. The calculated parameters were verified by direct optical observation under large magnification. While lateral size was 
a)

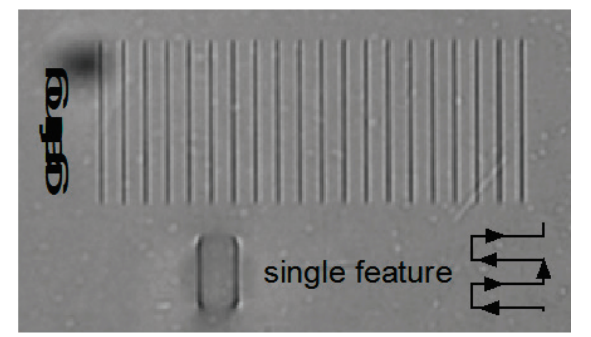

b)

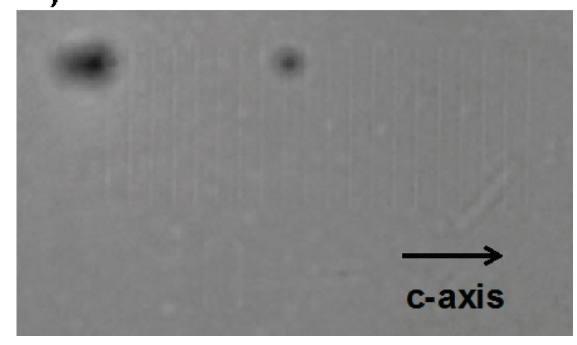

Fig. 5 Erasure of photorefractive structures recorded by DLW (a) the original recording features a grating and a larger rectangular area modified by scanning the laser beam along $c$-axis as shown by the arrows, (b) the same area of crystal after exposure by a defocused laser beam with a wavelength of $\lambda=532 \mathrm{~nm}$.

close to the calculated value, axial size was somewhat larger, approximately $60 \mu \mathrm{m}$, as is illustrated by side-view optical image shown in Fig. 4(a). While elongation of the focus limits the axial resolution of DLW, it may be helpful for fast fabrication of thick DOEs. Precise reason for such elongation is not clear at the moment, but is likely related to strong, spatially varying optical nonlinearity of photoexcited region of lithium niobate. In situations where focal elongation is not desired, it may be possible to suppress nonlinear effects using a recently proposed temporal focusing technique[13,14], which was successfully applied for reducing axial length of the focal region in DLW experiments. In this approach, a "spatial chirp" is introduced into the writing beam using a pair of dispersive elements (gratings or prisms), and various spectral components of the beam are forced to coincide spatially and temporally exclusively at the focus, resulting in spatially isotropic shape of the focal region.

Estimates of refractive index modulation were performed using measured thickness of the grating. Using the expressions given above, we have estimated refractive index modulation by an amount of $\Delta \mathrm{n} \approx 6 \times 10^{-4}$ at lowest recording laser power $(24 \mathrm{~mW})$ in Fig. 4(b). We note that this estimate was obtained for extraordinary probing beam polarized along the $c$-axis. For ordinary polarization (normal to $c$-axis) the modulation decreases approximately three times, reflecting lower electro-optical coefficient of lithium niobate along that direction. The estimated refractive index modulation is comparable to what was achieved previously in lithium niobate using other microfabrication techniques[8-11], and approaches previously determined saturation values[15] resulting in holographic recording experiments with increasing density of iron dopants. This limitation arises due to increase in conductivity at higher doping

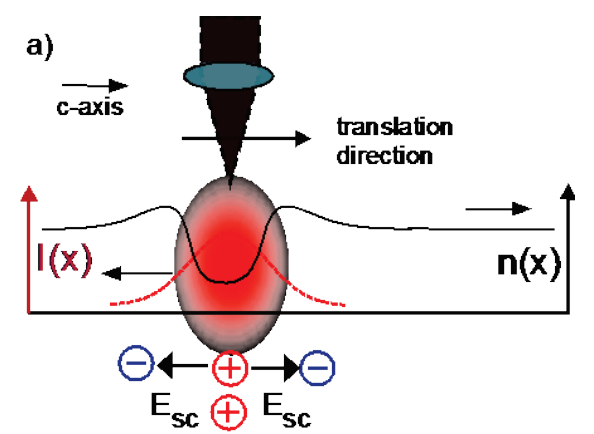

b)

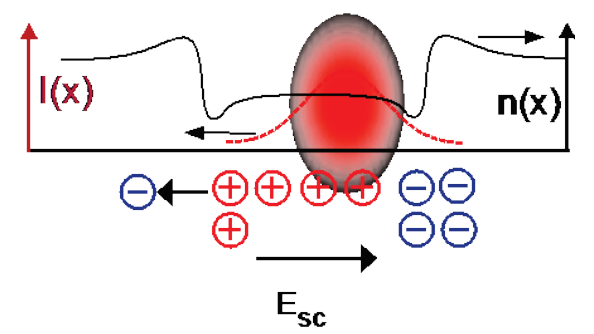

Fig. 6 The principle of local erasure of photorefractive structures by focus translation along $c$-axis. Laser intensity and photoinduced space charge for static focus (a), and moving focus (b).

levels, and known to increase photoconductivity[16]. Tunneling of electrons between closely-spaced iron dopants results in unwanted dark conductivity, which reduces lifetime of the recorded features.

Fig. 4(b) shows dependence of the first-order diffraction efficiency on the laser power. As can be seen, the efficiency tends to increase steeply at low laser powers, and saturates at higher powers. The saturation reflects depletion of optically active Fe donors by optical exposure.

\subsection{Optical erasure and re-writing}

Reversibility of optical recording means the possibility to erase and subsequently re-write micro-structures in the same volume of crystal. Photorefractive features decay naturally due to dark conductivity of lithium niobate, but this process is fairly slow, with time constant from days to months. Lifetime of the recording can be increased drastically using thermal fixing technique. Fast erasure of the entire crystal can be achieved using spatially uniform shortwavelength illumination. Figure 5(a) shows optical images of two structures (a grating and a rectangular region formed by a dense scan of the focus) immediately after the recording. To erase the structures, the crystal was irradiated by a frequency-doubled beam of a nanosecond $\mathrm{Nd}$ :YAG laser defocused to deliver irradiance of about $10 \mathrm{~mW} / \mathrm{cm}^{2}$. Optical contrast of microstructures decreases with time, and becomes barely visible after 40 minutes of exposure as shown in Fig. 5(b) before disappearing completely after $1 \mathrm{~h}$ exposure. Hence, permanent photomodification due to optical or thermal damage is absent and erasable photorefractive photomodification was induced during DLW.

In addition to global erasure of the entire crystal, it is possible to achieve local erasure using translation of the focus along the $c$-axis. This approach is explained schematically in Fig. 6. A stationary beam would create intensity distribution and refractive index modulation similar to 


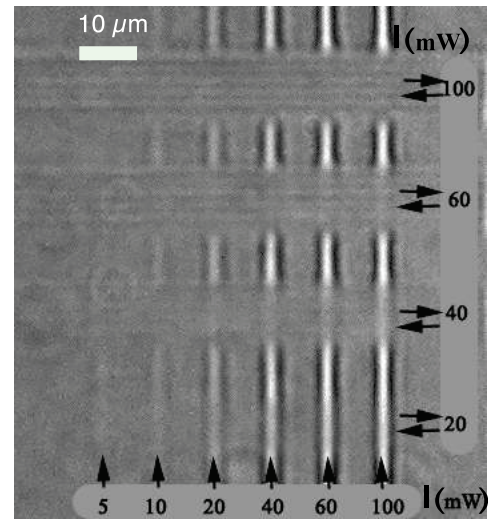

Fig. 7 Demonstration of global erasure by defocused laser beam, (a) original recording, (b) the same area of crystal after exposure by a defocused beam of a nanosecond laser with wavelength of $\lambda=532 \mathrm{~nm}$.

those shown in Fig. 6(a), with negative index change at the center, and positive changes in the periphery. If the focus is translated along the $c$-axis direction as shown in Fig. 6(b), new regions of the crystal will be photoexcited along the translation path. The photoexcited electrons will be pushed forward by the moving gradient of carrier density, leaving uncompensated ionized Fe dopants behind. As a result, nearly spatially uniform decrease of the refractive index, qualitatively similar to that shown in Fig. 6 will be induced. The translation will overwrite or strongly suppress index modulation of any previously recorded features. At the same time, photorefractive modification will be almost invisible for translation the $c$-axis, except for kinks at the start and stop points of the erasure line. This is similar to the previous demonstration obtained with single pulses of higher energy: refractive index contrast of single-shot exposure spots arranged along a line parallel to $c$-axis tends to disappear when separation between the spots becomes close to the spot size [9]. Thus, local erasure exploits optical anisotropy of lithium niobate. Unlike global optical or thermal erasure by spatially uniform exposure, which return crystal to its pre-recorded state, local erasure works by overwriting regions of crystal with optically invisible lines featuring spatially uniform negative refractive index change.

Practical demonstration of local erasure is presented in Fig. 7. First, vertical lines perpendicular to the $c$-axis were recorded at a constant translation velocity of $20 \mu \mathrm{m} / \mathrm{s}$ for various laser powers using NA $=0.35$ focusing lens. Subsequently, the recorded lines were partially erased by horizontal lines drawn parallel to the $c$-axis at the same velocity and for various laser powers. To erase wider regions, the erasure was done by sets of closely spaced lines separated by vertical distance of $\delta y=1.5 \mu \mathrm{m}$. As can be seen, optical contrast of the vertical lines (recording) becomes gradually suppressed with increasing exposure of the horizontal (erasure) lines.

Spatial distribution of the positive and negative charges shown in the qualitative scheme in Fig. 6(b) might be different from the actual one. However, our experimental data a)

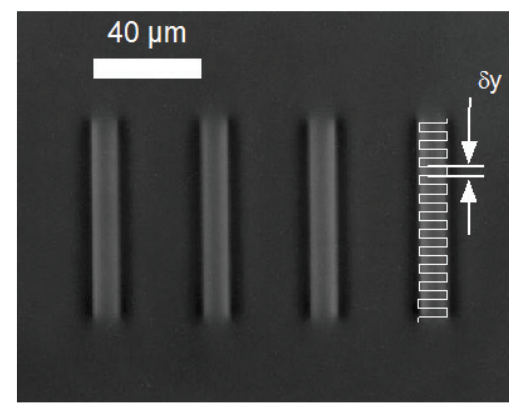

b)

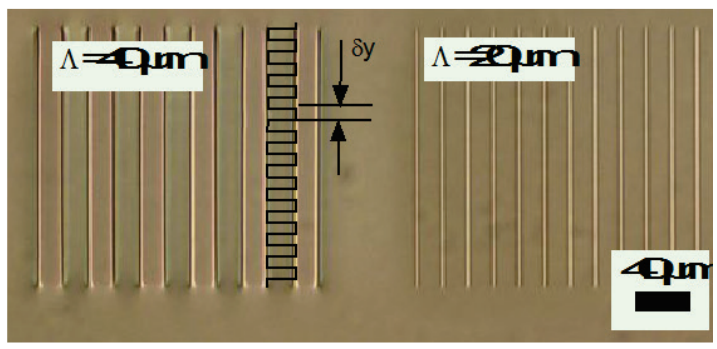

c)

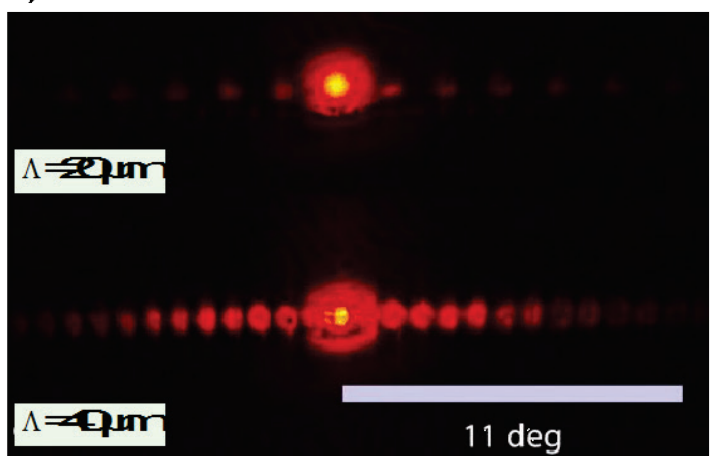

Fig. 8 Formation of binary refractive index modulation structures by DLW, (a) phase contrast microscopy image of rectangular stripe pattern, (b) square and line diffraction gratings and (c) their diffraction patterns.

confirms that nearly uniform decrease of the refractive index is induced by sweeping the focus in the c-axis direction. Fig. 8(a) shows phase contrast microscopy image of a structure comprised of $10 \mu \mathrm{m}$ wide vertical stripes. Each stripe consists of large number of closely-spaced horizontal lines recorded at a translation speed of $40 \mu \mathrm{m} / \mathrm{s}$ and a laser power of $25 \mathrm{~mW}$ using NA=0.4 focusing lens. The vertical scanning step was $0.2 \mu \mathrm{m}$. In the image, bright/dark regions correspond to negative/positive refractive index change. As can be seen, brightness of inner area of the stripes is fairly uniform. Intensity kinks near the sides of the stripes correspond to negative/positive refractive index modulation depicted previously in Fig. 6(b). Although one may expect that photosensitivity in the erased regions (e.g., inside the stripes) may become reduced, we have found this reduction insignificant, and the same region of crystal can be put through tens of write-erase cycles without significant decrease in the optical contrast of recorded features.

Local erasure (or writing along the c-axis) can be used to create patterns with quasi-binary index modulation profile, an impossible task for holographic recording. Fig. 7(b) shows optical images of two diffraction gratings. The first of them is a square grating with period of $\Lambda=40 \mu \mathrm{m}$ and 
stripe width of $20 \mu \mathrm{m}$, recorded by horizontal translation and dense vertical line scan explained schematically in the Figure. If dominant refractive index modulation in this grating would occur only near the edges of stripes, the grating would effectively consist of narrow lines, the grating period would be halved to $\Lambda=20 \mu \mathrm{m}$, and the diffraction angle would increase correspondingly. As a reference, another grating composed of single lines with lattice period $\Lambda=20 \mu \mathrm{m}$ was also recorded. Diffraction patterns of both gratings shown in Fig. 8(c) indicate clearly that the period halving has not occurred, and spatial profile of the first grating is rectangular, as expected.

\section{Conclusions}

Our results suggest that application of femtosecond DLW technique in lithium niobate can provide a highly sought combination of flexibility and reversibility of microstructuring of transparent optical materials. We have demonstrated fabrication of simple prototypes of DOE devices, and estimated the magnitude of photoinduced refractive index change. Clear advantage of DLW technique for microstructuring of lithium niobate is the capability of almost arbitrary 3D structuring in the bulk of the crystal. Precisely controlled translation of the writing beam can be used to realize local erasure of previously recorded features, and formation of structures with binary refractive index modulation profiles.

\section{References}

[1] K.K. Wong (ed.): EMIS datareview series, 28 (2002).

[2] K. Buse, A. Adibi, and D. Psaltis: Nature, 393, (1998) 665.

[3] L. Hesseling, S.S. Orlov, A. Liu, A. Akella, D. Lande and R.R. Neurgaonkar: Science, 282, (1998) 1089.

[4] V.M.N. Passaro, F. Magno: Laser Physics, 17 (2007), 231.

[5] A. Shiratori, R. Aida, R. Hagari, M. Obara: Jpn. J. Appl. Phys.: 37, (1998) L225.

[6] O. Beyer, I. Breunig, F. Kalkum, K. Buse: Appl. Phys. Lett., 88, (2006) 051120.

[7] W. Yang, P.G. Kazansky, Y.P. Svirko: Nature Photonics, 2 (2008) 99.

[8] D. Paipulas, V. Kudriašov, M. Malinauskas, V. Smilgevičius, V. Sirutkaitis: Appl. Phys. A, 104, (2011) 769-773.

[9] S. Juodkazis, V. Mizeikis and H. Misawa: J. Appl. Phys., 106, (2009) 51101.

[10] S. Juodkazis, M. Sudzius, V. Mizeikis, H. Misawa, E. G. Gamaly, Y. Liu, O. A. Louchev and K. Kitamura: Appl. Phys. Lett.,89, (2006) 062903.

[11]E. Gamaly, S. Juodkazis, V, Mizeikis, H. Misawa, A. Rode and W. Krolikowski: Phys. Rev. B, 81, (2010) 54113.

[12]R.J. Collier, C.B. Burkhardt, L.H. Lin: Optical Holography (Academic Press, San Diego, 1971), Ch. 9.

[13] F. He, H. Xu, Y. Cheng, J. Ni, H. Xiong, Z. Xu, K. Sugioka, K. Midorikawa, Opt. Lett., 35, (2010) 1106.
[14] D.N. Vetek, D. E. Adams, A. Johnson, P.S. Tsai, S. Backus, C.G. Durfee, D. Kleinfeld, J A. Squier, Opt. Express, 18, (2010) 18086.

[15]K. Peithmann, A. Wiebrock, K. Buse, Appl. Phys. B, 68, (1999) 777-784.

[16] I. Nee, M. Müller. K. Buse, E. Krätzig, J. Appl. Phys., $88,(2000)$ 4282-4286.

(Received: July 06, 2012, Accepted: October 02, 2012) 